\title{
Multidimensional light bullets in Fermi liquid in the presence of magnetic field and AdS/CFT correspondence
}

\author{
N. N. Konobeeva ${ }^{1}$, M. B. Belonenko ${ }^{1,2}$ \\ ${ }^{1}$ Volgograd State University, 400062, Volgograd, Russia \\ ${ }^{2}$ Laboratory of Nanotechnology, Volgograd Institute of Business, 400048, Volgograd, Russia \\ belonenko@volsu.ru, yana_nn@volsu.ru
}

PACS 71.10-Ay, 42.65. Re, 42.65. Tg

DOI 10.17586/2220-8054-2017-8-3-365-370

\begin{abstract}
We consider the evolution of a light bullet in a fermi-liquid while considering the presence of a magnetic field. The energy spectrum for the Fermi liquid was calculated in the framework of AdS/CFT correspondence. The influence of a magnetic field's amplitude and its period on light bullet evolution was obtained. The stable character of the light bullet propagation in the Fermi liquid in the presence of a magnetic field was confirmed.
\end{abstract}

Keywords: light bullets, AdS/CFT correspondence, magnetic field.

Received: 23 March 2017

Revised: 1 April 2017

\section{Introduction}

A theory for normal Fermi liquids was constructed by L.D. Landau in 1956 [1], based on the representation of the perturbation energy as a functional of the distribution function. This theory was further developed for finite Fermi systems by A. B. Migdal [2]. A Fermi liquid is a quantum fluid in which elementary excitations (quasiparticles) have a half-integral spin and obey Fermi-Dirac statistics (for example, conduction electrons in a metal).

The Fermi liquid theory continues to develop rapidly and it is used to solve modern problems in various fields of science. For example, in Ref. [3], based on the relativistic version of the Fermi liquid theory, an equation of state for the dense substance of a neutron star in the presence of a strong quantizing magnetic field was obtained. Another interesting research area is the study of non-Fermi and holographic Fermi liquids [4]. These schemes are based on charged black holes asymptotically inserted in Anti de Sitter (AdS) spaces. According to the AdS/CFT correspondence, quantum gravity on this background is holographically dual to certain field theories in flat space. When we add spinor fields to the gravitational theory, it is possible to realize holographic Fermi surfaces with a variety of Fermi liquid and non-Fermi liquid spectral functions [5].

In this paper, we investigate the influence of a Fermi liquid on light bullet propagation in the presence of a magnetic field. The dispersion law for the Fermi liquid is calculated based on the idea of AdS/CFT correspondence [6], which has recently found wide applications in solid state physics. This approach allowed us to obtain the Green's function [7,8], and to calculate the energy spectrum for the Fermi liquid.

Note, this approach has been well-proved and has already been used by researchers to study the evolution of extremely short optical pulses in a marginal Fermi liquid [9], which demonstrated the stable nature for the propagation of the one- and two-dimensional pulses due to the balance of the dispersion and the nonlinearity of the medium [9].

At the same time, the problem associated with the external magnetic field, remains unsolved.

\section{Basic equations}

We begin our investigation of the light bullet (LB) dynamics with well-known dispersion relation $E(p)$ for quasiparticles. At the construction of the LB propagation model, we describe the electromagnetic field of the pulse based on the Maxwell equations, in the Coulomb gauge [10] $\mathbf{E}=-\partial \mathbf{A} / c \partial t$. A vector-potential has a form: $\mathbf{A}=\left(0,0, A_{z}\right)$. In the two-dimensional (a) and three-dimensional case (b) we have (here the light velocity $c=1$ ):

$$
\begin{gathered}
\frac{\partial^{2} \mathbf{A}}{\partial x^{2}}+\frac{\partial^{2} \mathbf{A}}{\partial y^{2}}-\frac{\partial^{2} \mathbf{A}}{\partial t^{2}}+4 \pi j=0 \\
\frac{\partial^{2} \mathbf{A}}{\partial t^{2}}=\frac{1}{r} \frac{\partial}{\partial r}\left(r \frac{\partial A}{\partial r}\right)+\frac{\partial^{2} \mathbf{A}}{\partial x^{2}}+\frac{1}{r^{2}} \frac{\partial^{2} \mathbf{A}}{\partial \varphi^{2}}+4 \pi j=0,
\end{gathered}
$$


where $j$ is the electric current that appears due to the action of the electric field of the pulse on quasiparticles. Here, in the two-dimensional case, we neglect with the light bullet diffraction scattering in the direction perpendicular to the propagation axis. Since for the non-Fermi liquids, there is a region with a large lifetime of quasiparticles, the quasiparticles ensemble at typical time for the dynamics problems of the light bullets (order $10^{-14} \mathrm{~s}$ ) can be described in the framework of the collisionless kinetic Boltzmann equation [11]:

$$
\frac{\partial f}{\partial t}-\frac{q}{c} \sum_{\alpha} \frac{\partial A_{\alpha}}{\partial t} \frac{\partial f}{\partial p_{\alpha}}=0
$$

where $f=f\left(p_{\alpha}, s, t\right)$ is the distribution function, which implicitly depends on the coordinate. The distribution function $f$ at the initial time coincides with the equilibrium function of the Fermi distribution $F_{0}$ :

$$
F_{0}=\frac{1}{1+\exp \left\{E(\mathbf{p}) / k_{b} T\right\}},
$$

where $T$ is the temperature, $k_{b}$ is the Boltzmann constant. For the current density $\mathbf{j}=\left(0,0, j_{z}\right)$ we can write:

$$
j_{z}=q \int d^{3} p v_{z} f
$$

here, we introduce the group velocity $v_{z}=\partial E(\mathbf{p}) / \partial p_{z}$. Solving the equation (3) by the method of characteristics, one can obtain:

$$
j_{z}=q \int_{-q_{0}}^{q_{0}} \int_{-q_{0}}^{q_{0}} \int_{-q_{0}}^{q_{0}} d^{3} p v_{z}\left[p-\frac{q}{c} A_{z}(t)\right] F_{0}(\mathbf{p}) .
$$

The integration in Eq. (5) is carried out over the first Brillouin zone, $q_{0}$ corresponds to the layer near the Fermi surface. The dependence of the basic quantities on $q_{0}$ has a logarithmic nature, which allows us to arbitrarily choose it.

\section{The excitation dispersion law and AdS/CFT correspondence}

We begin with the dispersion law (here $k$ is measured from the Fermi level):

$$
\varepsilon=\varepsilon(|k|),|k|=\left(k_{z}^{2}+k_{\perp}^{2}\right)^{0.5} .
$$

It is more convenient to present in the following form:

$$
\varepsilon=\varepsilon\left(k_{z}, k_{\perp}^{2}\right) .
$$

Let us write the expression for the current density. It can be found, for example, in the Ref. [12]:

$$
j=n_{0} \int d k v_{x}\left(k_{z}-A, k_{\perp}^{2}\right) f\left(k_{z}, k_{\perp}^{2}\right),
$$

Here, $f$ is the distribution Fermi function, $v_{x}=\frac{\partial \varepsilon\left(k_{z}, k_{\perp}^{2}\right)}{\partial k_{x}}$.

For the low-temperature case, we can change $f$ on 1 in the layer near the Fermi surface. The layer thickness is defined with the conservation of the number of particles:

$$
\int_{-\Delta}^{\Delta} d k=\int_{Z B} d k f
$$

Then:

$$
j=n_{0} \int_{-\Delta}^{\Delta} d k_{z} \int_{0}^{\sqrt{2} \Delta} k_{\perp} d k_{\perp} \int_{0}^{2 \pi} d k_{\phi} \frac{\partial \varepsilon\left(k_{z}-A, k_{\perp}^{2}\right)}{\partial k_{x}}=\pi n_{0} \int_{0}^{2 \Delta^{2}} d x(\varepsilon(\Delta-A, x)-\varepsilon(-\Delta-A, x)) .
$$

Let us specify the dispersion law $\varepsilon=\varepsilon\left(k_{z}, k_{\perp}^{2}\right)$ using AdS/CFT correspondence ideas and following the work [13].

Let us consider two- and one-dimensional boundary theories, corresponding to a gravity theory in asymptotically $\mathrm{AdS}_{4}$. The Lagrangian can be chosen in standard type for both gravitational field and gauge field with U(1) symmetry [13]:

$$
L=\frac{1}{2 g^{2}}\left(R+\frac{6}{\Lambda^{2}}\right)-\frac{1}{4 e^{2}} F_{\mu \nu} F^{\mu \nu},
$$


where $g$ is gravitational interaction constant, $e$ is a charge, $F_{\mu \nu}$ is stress tensor for the gauge field. The given choice of the Lagrangian is in accordance with the solution as the Reissner-Nordstrom-AdS black hole:

$$
\begin{aligned}
d s^{2} & =\frac{\Lambda^{2}}{r^{2}}\left(-f(r) d t^{2}+\frac{d r^{2}}{f(r)}+d x^{2}+d y^{2}\right) \\
A_{0} & =\mu\left(1-\frac{r}{r_{+}}\right)
\end{aligned}
$$

here $\mu$ corresponds to chemical potential as usual, $r_{+}$is according to event horizon.

There are the following expressions:

$$
\begin{aligned}
f(r) & =1-\left(1+\frac{r_{+}^{2} \mu^{2}}{2 \gamma^{2}}\right)\left(\frac{r}{r_{+}}\right)^{3}+\frac{r_{+}^{2} \mu^{2}}{2 \gamma^{2}}\left(\frac{r}{r_{+}}\right)^{4}, \\
T & =\frac{1}{4 \pi r_{+}}\left(3-\frac{r_{+}^{2} \mu^{2}}{2 \gamma^{2}}\right), \\
\gamma & =e \Lambda / g,
\end{aligned}
$$

here $T$ is temperature. Selecting the Lagrangian for sample fermions in the following form:

$$
L=\bar{\psi} \Gamma^{\mu}\left(\partial_{\mu}+\frac{1}{4} \omega_{\mu}^{a b} \Gamma_{a b}+i A_{\mu}\right) \psi-m \bar{\psi} \psi
$$

where $m$ is mass, $\frac{1}{4} \omega_{\mu}^{a b} \Gamma_{a b}$ corresponds to spin interaction. Then, we define the Green's function for fermionic operator $\mathrm{O}_{\psi}$ as follows:

$$
G_{R}(t, \bar{x})=i \theta(t)\left\langle\left\{O_{\psi}(t, \bar{x}), O_{\psi}^{+}(0)\right\}\right\rangle
$$

where $\theta(t)$ is the Heaviside step function, angle brackets signify averaging, curved brackets signify anticommutator. As it is shown in [13] the Green's function has the form:

$$
G_{R}(w, \bar{k})=\frac{h}{k-k_{F}-w / v_{F}-\Sigma\left(w, k_{F}\right)}
$$

where $k=|\bar{k}|, k_{F}$ is Fermi momentum, $v_{F}$ is Fermi velocity. The main difference of the given Green's function, whose poles determine the dispersion law of quasiparticles lies in the fact that the quasiparticles' dissipation highly depends on parameter: $\nu_{k}=\frac{1}{\sqrt{6}} \sqrt{m^{2} L^{2}+\frac{3 k^{2}}{\mu^{2}}-\gamma^{2}}$. Note, $\nu$ is closely related to the critical exponent.

At low temperatures, the dispersion law can be written using AdS/CFT corrspondence:

$$
\varepsilon(k)=c\left(k_{z}^{2}+k_{\perp}^{2}\right)^{\nu / 2} .
$$

Taking into account the magnetic field, which we assume is spatially modulated for the problem generality: $H=Q \cdot \sin (\alpha \cdot y)$, we can write the current density in its explicit form:

$$
\begin{gathered}
j(A)=\frac{\pi n_{0} c}{0.5 \nu+1}\left((\Delta+A)^{\delta_{1}}-(\Delta-A)^{\delta_{1}}+\left((\Delta-A)^{2}+2 \Delta^{2}\right)^{\delta_{2}}-\left((\Delta+A)^{2}+2 \Delta^{2}\right)^{\delta_{2}}\right), \\
\delta_{1}=\nu+2+H, \quad \delta_{2}=0.5 \nu+1+H .
\end{gathered}
$$

The relation between the parameter $\nu$ and the magnetic field is given by formula [14]:

$$
v_{k}=\sqrt{\frac{k^{2}+m^{2}}{6}-\frac{q_{e f f}^{2}}{12}}, \quad q_{e f f}=q \sqrt{1-\frac{h^{2}}{3}},
$$

where $q$ is the fermion charge, $q_{e f f}$ is the effective charge, $h$ is the magnetic field, $m$ is the fermion mass.

\section{Dynamics of light bullets with a plane wave front}

Further, we consider two-dimensional light bullets with the initial conditions:

$$
\begin{aligned}
A(x, y, 0) & =B \cdot \exp \left(-x^{2} / \gamma_{x}^{2}\right) \cdot \exp \left(-y^{2} / \gamma_{y}^{2}\right), \\
\frac{d A(x, y, 0)}{d t} & =B \cdot \frac{2 u}{\gamma_{x}^{2}} \cdot \exp \left(-x^{2} / \gamma_{x}^{2}\right) \cdot \exp \left(-y^{2} / \gamma_{y}^{2}\right),
\end{aligned}
$$


where is the pulse amplitude, $u$ is the initial velocity of the pulse along the $x$ axis, $\gamma_{x}, \gamma_{y}$ define the pulse width. Equations $(1,14)$ were solved numerically with a direct difference scheme of the cross type [15]. The time and coordinate steps were determined from the standard stability conditions.

The influence of the magnetic field amplitude on two-dimensional light bullets is presented in Fig. 1.
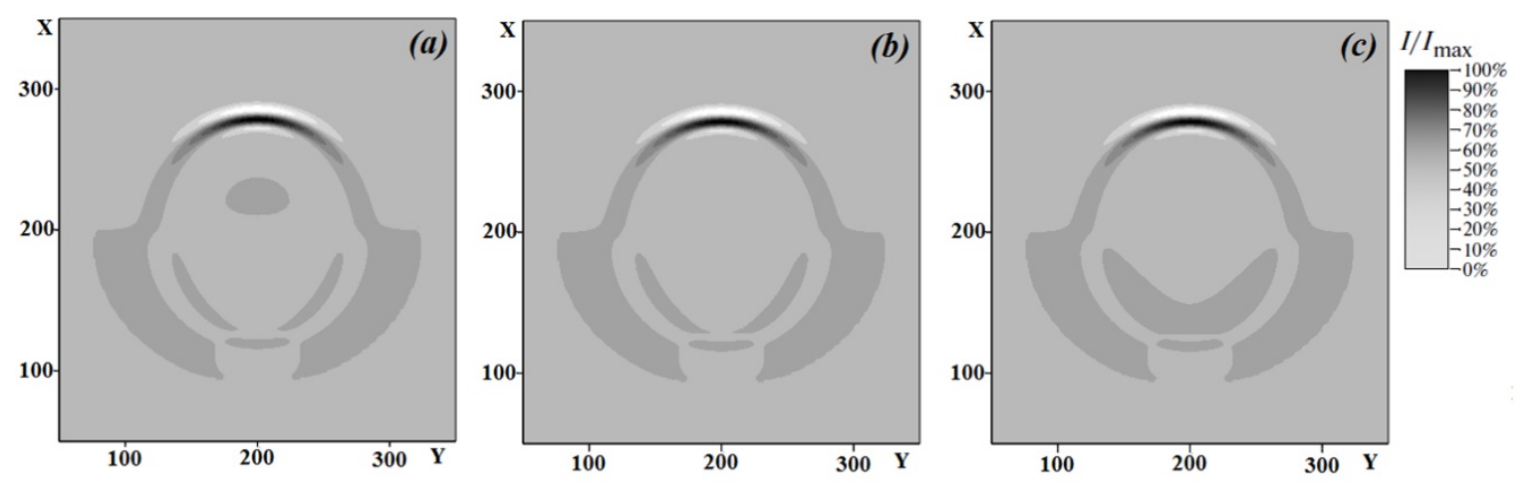

FIG. 1. The intensity of 2D electromagnetic pulse $I(x, y, t)=E^{2}(x, y, t)$ for the Fermi liquid $\left(t=1 \cdot 10^{-13} \mathrm{~s}, \alpha=0.01\right)$ : a) $Q=0.1$; b) $Q=0.2$; c) $Q=0.4$. Units along the $x$ and $y$ axis correspond to $20 \mathrm{~nm}$

It should be noted that the magnetic field amplitude practically has little effect on the shape and amplitude of the main pulse. We have only changes in the "tail" after the main pulse. Moreover, the larger Q value, the greater part of the energy is pumped to the "tail".

The influence of the period of modulation of the magnetic field on a two-dimensional ultimately short pulse is demonstrated in Fig. 2.
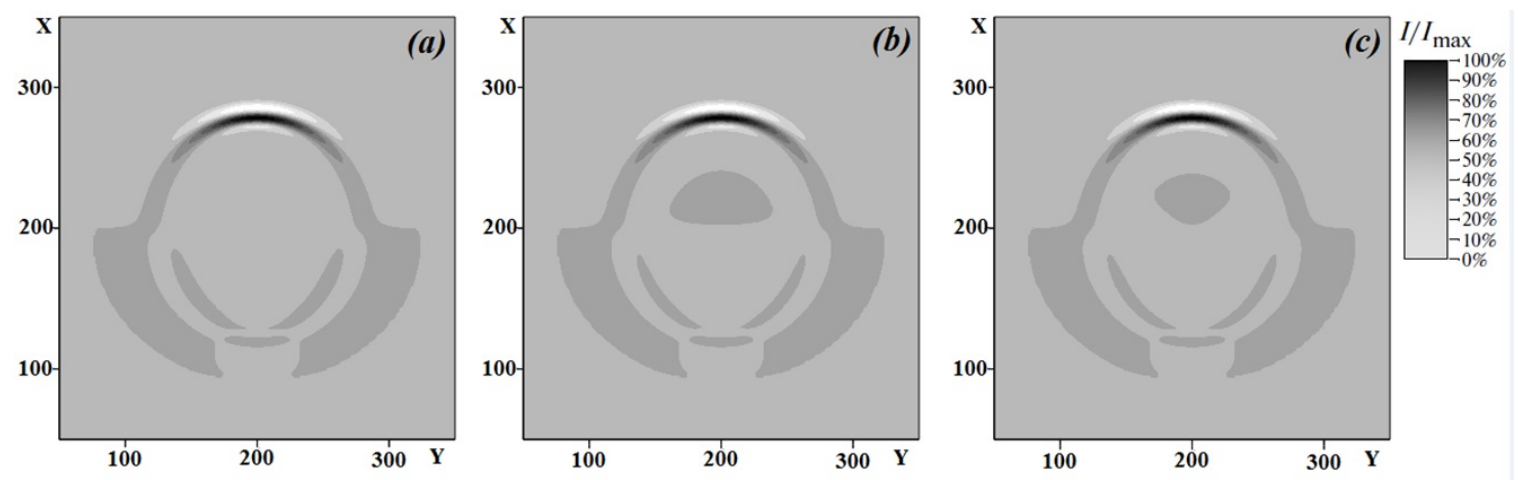

FIG. 2. The intensity of $2 \mathrm{D}$ electromagnetic pulse $I(x, y, t)=E^{2}(x, y, t)$ for the Fermi liquid $\left(t=1 \cdot 10^{-13} \mathrm{~s}, Q=0.2\right)$ : a) $\alpha=0.01$; b) $\alpha=0.05$; c) $\alpha=0.1$. Units along the $x$ and $y$ axis correspond to $20 \mathrm{~nm}$

As in the case of different amplitude values for the magnetic field, we observe a change in the shape of the "tail" following the main pulse, which is not affected by the period of the magnetic field.

A picture of the light bullet propagation in the Fermi liquid in the three-dimensional case can be seen in Fig. 3 and Fig. 4. The initial condition in the three-dimensional case was chosen in the form:

$$
\begin{aligned}
A(x, r, 0) & =R \cdot \exp \left(-\frac{\left(x-x_{0}\right)^{2}}{\gamma_{x}^{2}}\right) \exp \left(-\frac{r^{2}}{\gamma_{r}^{2}}\right), \\
\frac{d A(x, r, 0)}{d t} & =2 R \nu_{x} \frac{\left(x-x_{0}\right)}{\gamma_{x}^{2}} \exp \left(-\frac{\left(x-x_{0}\right)^{2}}{\gamma_{x}^{2}}\right) \exp \left(-\frac{r^{2}}{\gamma_{r}^{2}}\right),
\end{aligned}
$$

where $r$ is the radius, $R$ is the amplitude, $\gamma_{x}, \gamma_{r}$ define the pulse width, $x_{0}$ is the initial displacement of the pulse center, $\nu_{z}$ is the initial velocity of the pulse along the $x$ axis. Note that as in the $2 \mathrm{D}$ case, time is the evolution variable. 

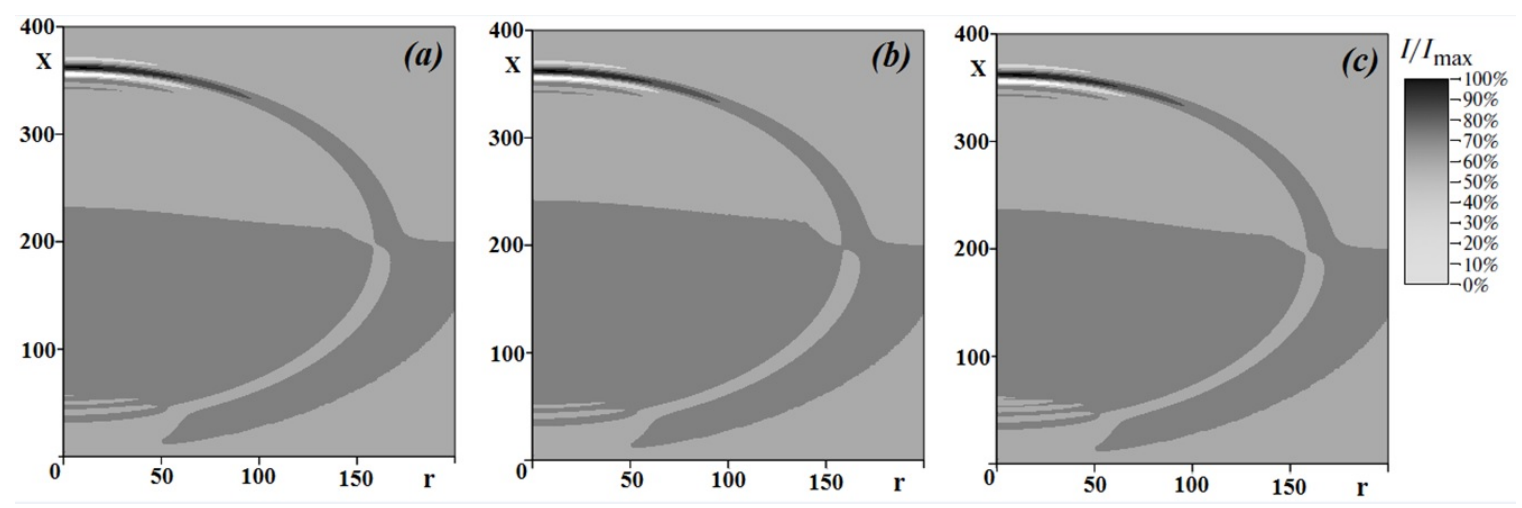

FIG. 3. The intensity of 3D electromagnetic pulse $I(x, r, t)=E^{2}(x, r, t)$ for the Fermi liquid $\left(t=1 \cdot 10^{-13} \mathrm{~s}, \alpha=0.01\right)$ : a) $Q=0.1$; b) $Q=0.2$; c) $Q=0.4$. Unity along the $r$-axis corresponds to $30 \mathrm{~nm}$, along the $x-20 \mathrm{~nm}$
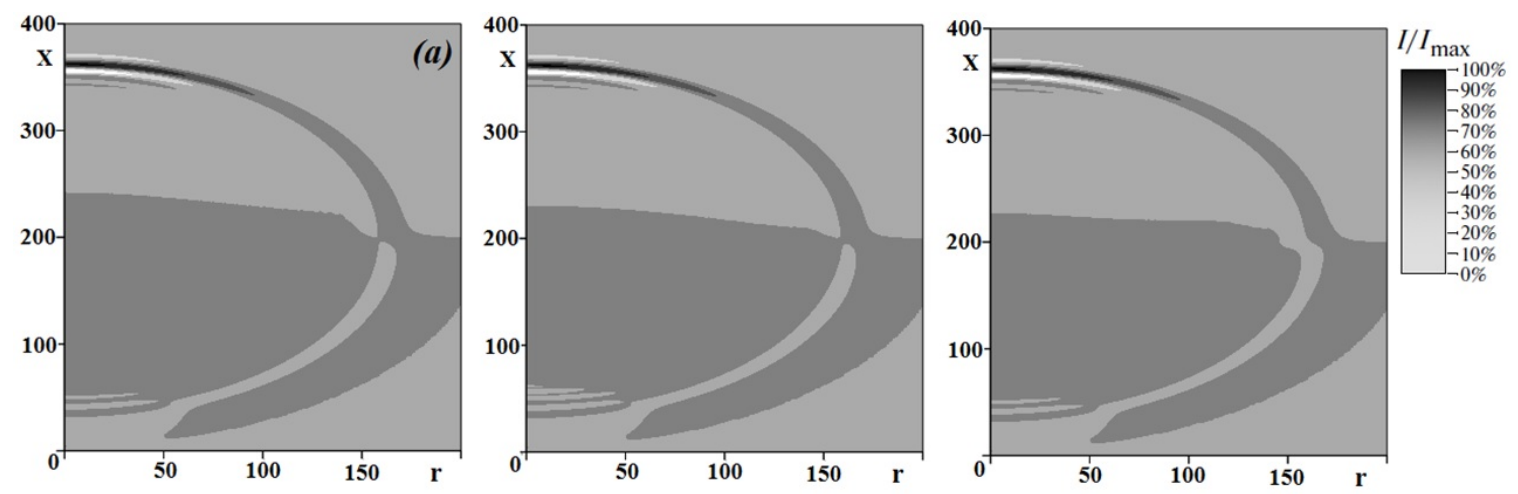

FIG. 4. The intensity of 3D electromagnetic pulse $I(x, y, t)=E^{2}(x, y, t)$ for the Fermi liquid $\left(t=1 \cdot 10^{-13} \mathrm{~s}, Q=0.2\right)$ : a) $\alpha=0.01$; b) $\alpha=0.05$; c) $\alpha=0.1$. Unity along the $r$-axis corresponds to $30 \mathrm{~nm}$, along the $x-20 \mathrm{~nm}$

Here, we observe an analog of the two-dimensional pulse behavior. And, accordingly, the change in the amplitude and period of the magnetic field causes only a change in the "tail" shape.

Thus, it is shown that the light bullet in the Fermi liquid propagates stably when the main parameters of the modulated magnetic field (amplitude and period) change.

\section{Conclusions}

Based on the obtained results, we can conclude the following:

1. The model, which describes the dynamics of ultra-short $2 \mathrm{D}$ and $3 \mathrm{D}$ laser pulses in a Fermi liquid in the frameworks of AdS/CFT correspondence while taking into account the magnetic field, is proposed.

2. The numerical calculations show that in the $2 \mathrm{D}$ and $3 \mathrm{D}$ cases, there are stable nonlinear waves, light pulses that are localized in two or three directions.

3. Regarding the light bullet propagation in the Fermi liquid, its dispersion in the transverse direction is rather weak, and the energy is mainly concentrated in the central region of the pulse.

\section{Acknowledgments}

This work was supported by the Ministry of Education and Science of the Russian Federation, Project No. MK4562.2016.2 and by the Russian Foundation for Basic Research (Project No. 16-47-340366). Numerical modeling was carried out in the framework of the state assignment of the Ministry of Education and Science (Project No. 2.852.2017/4.6). 


\section{References}

[1] Landau L.D. The theory of a Fermi liquid. JETP, 1956, 30(7), P. 1058-1064.

[2] Midgal A.B. The theory of finite Fermi systems and properties of atomic nuclei. Nauka, Moscow, $1983,432 \mathrm{p}$.

[3] Mandal S., Saha R., Ghosh S., Chakrabarty S. Neutron star equation of state and the possibility of complex self-energy in Landau theory of a Fermi liquid in the presence of a strong, quantizing magnetic field. Phys. Rev. C, 2006, 74, P. 015801.

[4] Sachdev S. What can gauge-gravity duality teach us about condensed matter physics? Ann. Rev. Condensed Matter Phys., 2012, 3, P. 9-33.

[5] Faulkner T., Iqbal N., Liu H., McGreevy J., Vegh D. Strange metal transport realized by gauge/gravity duality. Science, 2010, 329, P. 1043-47.

[6] Belonenko M.B., Konobeeva N.N., Galkina E.N. Quick introduction into AdS/CFT correspondence in physics of strongly correlated systems. Nanosystems: physics, chemistry, mathematics, 2016, 7(4), P. 410-421.

[7] Maldacena J.M. The large N limit of superconformal field theories and supergravity. Int. J. Theor. Phys., 1999,38, P. $1113-1133$.

[8] Pal Sh.S. Model building in AdS/CMT: DC conductivity and Hall angle. Phys Rev D., 2011, 84, P. 126009.

[9] Konobeeva N.N., Belonenko M.B. Propagation of few cycle optical pulses in marginal Fermi liquid and AdS/CFT correspondence Physica B: Condensed Matter, 2015, 478, P. 43-46.

[10] Yanyushkina N.N., Beloneko M.B., Lebedev N.G. Amplification of ultimately-short pulses in graphene in the presence of a high-frequency field. Optics and spectroscopy, 2010, 108 (5), 618-623.

[11] Kryuchkov S.V. Semiconductor superlattices in strong fields [in Russian], Peremena, Volgograd, 1992,67 p.

[12] Konobeeva N.N., Belonenko M.B. Dynamics of Ultimately Short Electromagnetic Pulses in Silicene Waveguides. Technical physics letters, 2013, 39, P. 579-581.

[13] Benini F. Holography and condensed matter. Fortschr. Phys. 2012, 60, P. 810-821.

[14] Basu P., Yang He J., Mukherjee A., Shieh H.-H. Holographic non-Fermi liquid in a background magnetic field. Phys. Rev. D., 2010, 82, P. 044036.

[15] Bahvalov N.S. Numerical methods (analysis, algebra, ordinary differential equations). Nauka, Moscow, 1975.632 p. 